\title{
Segment III bypass: An Option to Palliate Proximal Biliary Obstruction
}

\author{
Ramesh Singh Bhandari \\ Associate Professor, Department of GI and General Surgery, Tribhuvan University Teaching Hospital, Kathmandu, Nepal \\ Correspondence: Dr Ramesh Singh Bhandari, Department of GI and General Surgery, Tribhuvan University Teaching \\ Hospital, Kathmandu, Nepal.
}

Email: rsbhandari09@gmail.com

\begin{abstract}
Introduction: In the era of minimally invasive therapy, endoscopic or interventional radiological techniques have become preferred technique for palliating advanced malignant hilar obstruction due to carcinoma gallbladder or cholangiocarcionmas. However, in selected group of these locally advanced unresectable disease and fit patients, surgical segment III bypass could still be a viable option for palliation.
\end{abstract}

Methods: I retrospectively reviewed the medical records of all the patients of segment III bypass performed over last six years (Oct 2012- May 2018) and analyzed the indication, perioperative morbidity, symptom relief and survival.

Results: Total 17 patients underwent palliative biliary bypass over the specified period. Among them 8 patients underwent segment three III biliary bypass for proximal biliary obstruction. Except 1 patient, all segment three bypass were offered to malignant proximal biliary obstruction. One patient developed postoperative sepsis and had mortality on day 7. Symptom relief in the form of relief of pruritus, improved sleep pattern and improved appetite was seen in 5 patients. In remaining 2 patients, the bilirubin didn't drop to normal level and the symptom reliefs were partial. However, the patient had good satisfaction over not having an external tube attached to their body. Despite the patent anastomosis, failure in dropping bilirubin level significantly was considered due to segmental obstructions. One patient survived for 18 months, 1 had follow up to 13 months. Three patients were lost to follow up after 3 months. Two patients had six weeks follow up and were symptomatically doing better.

Conclusion: Surgical segment III bypass is still a good option for palliating malignant hilar obstruction in selected group of patients.

Keywords: Malignancy; Hilar obstruction; Segment III bypass.

\section{Introduction}

Majority of patients with malignant hilar obstructions either due to gall bladder cancers or hilar cholangiocarcinomas present in unresectable stages requiring some form of palliation. ${ }^{1-3}$ In the current era, endoscopic or radiology guided stenting procedures are considered as standard procedure for palliating malignant obstructive jaundice. ${ }^{4}$
However, even in tertiary level hospitals of developing nations, advanced endoscopic services may not be available. Sometimes the cost of endoscopic procedures outweighs the advantages of these procedures while palliating malignant obstructive jaundice. Endoscopic or radiology guided stenting procedures are not devoid of complications. Stent 
dislodgement and blockages are known complications. This requires repeated hospital admission and stent exchange procedures and thus increasing the cost of palliation. ${ }^{5}$ Surgical bypass for malignant obstruction, associated with increased morbidity in the beginning has been found more cost effective in long run when compared with stenting procedures. ${ }^{5}$ Biliary bypass for distal obstruction has remained a standard procedure while there are very limited reports regarding the palliative biliary bypass for proximal obstruction. Here, we intend to discuss our experience with segment III bypass offered to palliate patients with proximal biliary obstruction.

\section{Methods}

Retrospective review of the medical records of all the patients with proximal biliary obstruction who had undergone segment III bypass performed by single unit at Tribhuvan University Teaching Hospital, between Oct 2012- May 2018 were reviewed. Patient's demographics, Indications of surgery, duration of hospital stay, perioperative outcome and improvement in terms of fall in bilirubin and symptom relief (most importantly pruritus) were evaluated.

All patients were initially extensively worked up with different blood test and imaging test to find out resectability. Unresectability was confirmed on triphasic hepatobiliary MDCT imaging. MRCP was preformed selectively in patients who didn't undergo percutaneous biliary drainage (PTBD) and CT images were not clear to asses the hilar confluence anatomy. Locally advanced, unresectable but non metastatic disease and medically fit patients were given an option to opt either the stenting procedures, continue PTBD drainage or undergo palliative segment III bypass. Those who had opted for surgical bypass were operated under general anesthesia (GA) with right upper quadrant reverse L or hockey stick incision. Segment III duct was approached to the left side of the falciform ligament (Figure 1). Sometimes some parts of liver tissue was also incised to access the left duct. Duct was confirmed by aspiration of bile with insulin syringe after which the duct was wide opened (Figure 1). Roux limb of Jejunum was prepared, a small enterotomy was made and side-to-side, mucosa-to-mucosa bilioenteric anastomosis was made with 50 or 60 prolene suture (Figure 2). If the stent was already present, it was left as it is and removed postoperatively after a cholangiogram obtained through that the PTBD tube. Otherwise no stent was placed. A single tube drain $24 \mathrm{~F}$ size was placed at the anastomotic site, which was removed postoperatively when there was no clinical relevant drain output. All patients received standard postoperative management including a course of antibiotics on the basis of bile culture sensitivity reports.

\section{Results}

Total 19 patients underwent palliative biliary bypass over the specified period. Among them 11 patients were of distal biliary obstruction and underwent single or double bypass while remaining 8 patients underwent segment three III biliary bypass for proximal biliary obstruction. Except 1 patient, all segment three bypass were offered to malignant proximal biliary obstruction (Table 1). One patient with benign bile duct stricture was planned for Hepp- Couinad left duct hepaticojejunostomy but because the access was difficult due to severe adhesions in the hilum, segment three duct was approached for bypass.

All patients were operated with left upper quadrant reverse $\mathrm{L}(\mathrm{n}=5)$ or hockey stick incision $(\mathrm{n}=3)$. Patients who were opened with an intention to undergo radical surgery for malignancy were explored with reverse L incision and those who were preoperatively decided for segment III bypass were accesed with hockey stick incisions. Total 4 patients with carcinoma gallbladder and 1 with cholangiocarcinoma were explored with possibility of radical surgery. Unresectablity due to locally advanced disease was confirmed intraoperatively and segment III bypass was performed. One patient was of benign biliary stricture while in remaining two patients it was preoperatively planned for segment three bypass. Total 6 patients had preoperative PTBD drainage, 1 had endoscopic stenting and 1 had no preoperative drainage. All patients who had preoperative drainage had evidence of at least one episode of cholangitis and had improved with drainage and antibiotics.

Postoperative outcomes (Table 2) were assessed in terms of symptom relief and fall in bilirubin. One patient developed postoperative sepsis and had mortality on day 7 . Cholangiogram was performed in 6 patients having external drainage and with confirmation of patent bilioenteric anastomosis; the tube was clamped and removed after 48hours. Symptom relief in the form of relief of pruritus, improved sleep pattern and improved appetite was seen in at least 5 patients. In remaining 2 patients, the bilirubin didn't drop to normal level and the symptom reliefs were partial. However, the patient had good satisfaction over not having an external tube attached to their body. Despite the patent anastomosis, failure in dropping bilirubin level significantly was considered due to segmental obstructions. One patient survived for 18 months, 1 had follow up to 13 months. Three patients were lost to follow up after 3 months. Two patients had six weeks follow up and were symptomatically doing better. 
Table 1. Patient's demographics

\begin{tabular}{|c|c|}
\hline Parameters & Frequency $(n=8)$ \\
\hline $\begin{array}{l}\text { Female: Male } \\
\text { Indications }\end{array}$ & $6: 2$ \\
\hline Carcinoma Gallbladder & 5 \\
\hline Hilar Cholangiocarcinoma & 2 \\
\hline Benign Biliary stricture & 1 \\
\hline PTBD prior to surgery & 6 \\
\hline Endoscopic stenting & 1 \\
\hline
\end{tabular}

Table 2. Postoperative outcome

Post operative outcome
$\begin{aligned} & \text { Parameters } \\ & \text { Symptom improvement at } 6\end{aligned}$
weeks

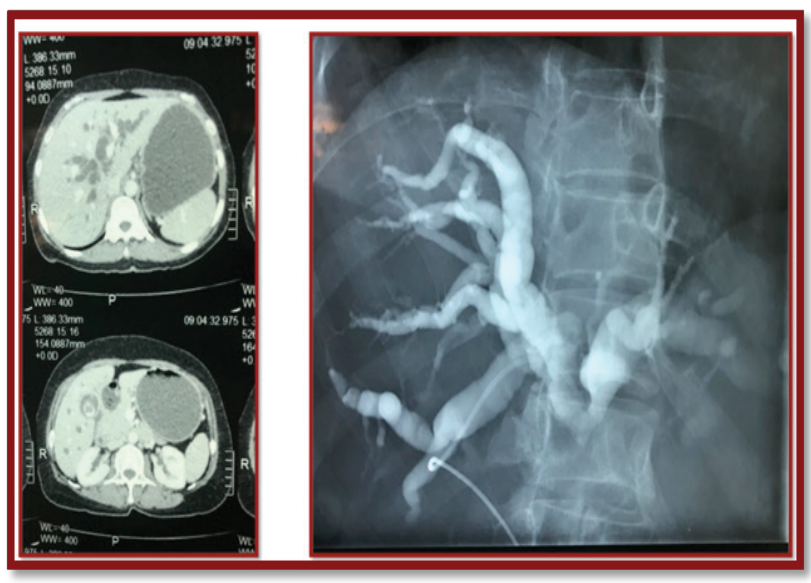

Figure 1. Hilar obstruction due to carcinoma Gallbladder
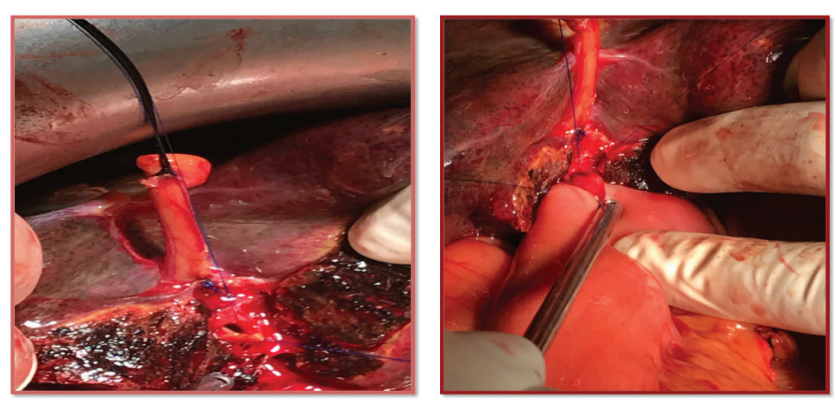

Figure 2. Segment three-duct and bilioenteric anastomosis

\section{Discussion}

In our series, we had performed segment three surgical bypass in 8 patients with hilar obstruction among which 7 were suspected malignant obstruction (either hilar cholangiocarcinoma or gallbladder carcinoma) and one was for benign biliary stricture. The decrease in level of jaundice and symptoms relief by six weeks in $80 \%$ of patients was very encouraging in terms of palliating the patients with malignant hilar obstruction. We have noticed that majority these patients come from middle to low-income groups and from very distant palces. For many of these patients, cost of stenting would be much higher than the surgery and also considering risk of tube blockage and dislodgements requiring repeated admissions the cost of surgery would further be less than non-surgical options. So, considering all these issues, the surgical bypass in selected group of patients could offer one time cost effective palliation.

In a review of 83 patients of unresectable hilar cholangiocarcinoma, two groups one with surgical bypass and the other with non-surgical treatments in the form of percutaneous drainage were compared. ${ }^{5}$ Demographic data, perioperative complication and late complications were comparable in two groups. However, median survival time was more with surgical bypass group (160 days versus 82 days.). Another review article published also reviewed two retrospective studies by lai et al and Pichlmayr et al. ${ }^{4}$ Both studies compared percutaneous or endoscopic approach with surgical bypass. Overall survival was more in Lai et al study (3.03 months Versus 1.46 months) while it was similar in Pichlmayr study. ${ }^{6,7}$ However, the perioperative mortality was higher in surgical group.

Besides these report there aren't many literature available comparing surgical bypass with non-surgical palliation either percutaneous approach or endoscopic approach. However, there are plenty of evidences available to support the use of non-surgical treatment options in palliating 
unresectable hilar obstruction. In the current era, whenever available and feasible endoscopic or percutaneous approach is preferred by most. Additionally, these procedures being minimally invasive have less perioperative morbidity. ${ }^{4}$ But those limited studies have shown better long-term survival with the surgical bypass. ${ }^{5}$

The median survival time for unresectable proximal biliary obstruction is around three months without any intervention palliation increase survival as well. ${ }^{8-10}$ These days surgical segment three bypass could be preferred options in situations like locally advanced disease but nonmetastatic in a very fit patient who is also opting for surgical over endoscopic treatment. Most ideal situation to perform segment three bypass would be in a condition where patient is explored with intention to undertake radical surgery but unresectability is detected intraoperatively. ${ }^{11}$ Because of the advancement in radiological and endoscopic technique, the role of surgical bypass might seem to be very limited. However, but in countries like ours where these endoscopic and radiological facilities are not easily available at most centers and also patients come from distant places for whom repeated hospital visits turn out to be more expensive and very difficult, Surgical Segment three bypass could offer them one time cost effective palliation with good long term palliation however at the cost increased perioperative morbidity.

\section{Conclusion}

The radiological or endoscopic techniques are the preferred techniques of palliation for malignant hilar obstruction like in locally advanced gallbladder carcinoma or hilar cholangiocarcinomas. However, in very selected group of patients and in developing countries with limited resources and repeated hospital visits become an issue for patients, segment three bypass could still be an option for palliating these locally advanced unresectable malignancies.

\section{References}

1. Mulholland MW, Yahanda A, Yeo CJ. Multidisciplinary management of perihilar bile duct cancer. J Am Coll Surg 2001; 193: 440-7. https://doi.org/10.1016/S10727515(01)01029-8

2. Jarnagin WR, Fong Y, DeMatteo RP, Gonen M, Burke EC, Bodniewicz BJ, et al. Staging, resectability, and outcome in 225 patients with hilar cholangiocarcinoma. Ann Surg 2001; 234: 507-17. https://doi.org/10.1097/00000658-200110000-00010 PMid:11573044 PMCid:PMC1422074
3. Reed DN Jr, Vitale GC, Martin R, Bas H, Wieman TJ, Larson GM, et al. Bile duct carcinoma: trends in treatment in the nineties. Am Surg 2000; 66: 711-4. PMid:10966023

4. H.Witzigmann,HLang,HLauer.Guidelinesforpalliative surgery of cholangiocarcinoma. HPB, 2008; 10: 154160. https://doi.org/10.1080/13651820801992567 PMid:18773044 PMCid:PMC2504365

5. Narongchai Wongkonkitsin, sêpiAke Phugkhem , Kriangsak Jenwitheesuk, O-Tur Saeseow, Vajarabhongsa Bhudhisawasdi. Palliative Surgical Bypass versus Percutaneous Transhepatic Biliary Drainage on Unresectable Hilar Cholangiocarcinoma. J Med Assoc Thai 2006; 89 (11): 1890-5. PMid: 17205870

6. Pichlmayr R, Weimann A, Klempnauer J, et al. Surgical treatment in proximal bile duct cancer. A single center experience. Ann Surg 1996;224:628 38. https://doi.org/10.1097/00000658-199611000-00007 PMid:8916878 PMCid:PMC1235440

7. Lai ECS, Chu KM, Lo CY, Fan ST, Lo CM, Wong J. Choice of palliation for malignant hilar biliary obstruction. Am J Surg 1992;103:208. https://doi. org/10.1016/0002-9610(92)90102-W

8. Hemming AW, Reed AI, Fujita S, et al. Surgical management of hilar cholangiocarcainoma. Ann Surg 2005;241:693-9; discussion 699-702. https:// doi.org/10.1097/01.sla.0000160701.38945.82 PMid:15849505 PMCid:PMC1357124

9. Farley DR, Weaver AL, Nagorney DM. "Natural history" of unresected cholangiocarcinoma: patient outcome after non- curative intervention. Mayo Clin Proc 1995;70:425-9. https://doi.org/10.4065/70.5.425 PMid:7537346

10. Kawasaki S, Imamura H, Kobayashi A, et al. Results of surgical resection for patients with hilar bile duct cancer: application of extended hepatectomy after biliary drainage and hemihepatic portal vein embolization. Ann Surg 2003; 238:84-92. https:// doi.org/10.1097/01.SLA.0000074984.83031.02 PMid:12832969 PMCid:PMC1422661 